Article

\title{
Local Coordination Environment of 3d and 4d Transition Metal Ions in LiCl-KCl Eutectic Mixture
}

\author{
Jon Fuller ${ }^{1,2} \mathbb{D}$, William Phillips ${ }^{1}$, Qi An ${ }^{2, * \mathbb{D}}$ and Ruchi Gakhar ${ }^{1, *}$ \\ 1 Idaho National Laboratory, Pyrochemistry and Molten Salt Systems Department, Idaho Falls, ID 83415, USA; \\ jonfuller@nevada.unr.edu (J.F.); william.phillips@inl.gov (W.P.) \\ 2 Department of Chemical and Materials Engineering, University of Nevada, Reno, NV 89577, USA \\ * Correspondence: qia@unr.edu (Q.A.); ruchi.gakhar@inl.gov (R.G.)
}

Citation: Fuller, J.; Phillips, W.; An, Q.; Gakhar, R. Local Coordination

Environment of $3 \mathrm{~d}$ and $4 \mathrm{~d}$ Transition Metal Ions in LiCl-KCl Eutectic Mixture. Materials 2022, 15, 1478. https://doi.org/10.3390/ma15041478

Academic Editor: Olga Russina

Received: 22 December 2021

Accepted: 9 February 2022

Published: 16 February 2022

Publisher's Note: MDPI stays neutral with regard to jurisdictional claims in published maps and institutional affiliations.

Copyright: (C) 2022 by the authors. Licensee MDPI, Basel, Switzerland. This article is an open access article distributed under the terms and conditions of the Creative Commons Attribution (CC BY) license (https:/ / creativecommons.org/licenses/by/ $4.0 /)$.

\begin{abstract}
In this study, the structure and coordination environment of two $3 \mathrm{~d}$ transition elements ( $\mathrm{Ni}$ and $\mathrm{Cr}$ ) is investigated in a molten chloride salt system. Electronic absorption spectroscopy was employed to elucidate their coordination environment in $3 \mathrm{LiCl}-2 \mathrm{KCl}$ eutectic salt, as a function of temperature. Density functional theory (DFT) modeling was used to determine the coordination environment of the transition metal species in the eutectic composition as well as the optical spectra computationally. The $\mathrm{Ni}^{2+}$ and $\mathrm{Cr}^{3+}$ exist in a tetrahedral and octahedral coordination environment, respectively, in eutectic salt. The spectra thus obtained were compared with the experimental data; a reasonable qualitative agreement was obtained between experimental and computational $\mathrm{Ni}^{2+}$ and $\mathrm{Cr}^{3+}$ spectra, and the coordination of both elements in the eutectic composition were in excellent agreement with the experimentally determined results. Computational results were also obtained for two $4 \mathrm{~d}$ elements, $\mathrm{Mo}^{3+}$ and $\mathrm{Nb}^{3+}$, with both quantum molecular dynamics (QMD) and hybrid functional optical spectra indicating octahedral coordination.
\end{abstract}

Keywords: HSE; DFT; molten salts; LiCl-KCl; optical absorption spectroscopy

\section{Introduction}

The coordination chemistry of various metal species in different molten salt compositions has become a very important focus for research development in molten salts, in order to discover the nature of their chlorocomplex formation mechanisms and their relationships with chemical processes, which are relevant to the functionality of molten salts applications, such as corrosion, electrolysis effects and other chemical processes [1,2]. Particularly, molten salt-based pyroprocessing technology is the key process for treating the used nuclear fuels to close the nuclear fuel cycle [3]. The thermodynamic and electrochemical properties of actinides and other fission products in eutectic molten $\mathrm{LiCl}-\mathrm{KCl}$ salt are pivotal to optimize, monitor and control the pyroprocessing process [4]. The coordination chemistry of these metal complexes in the melt are intrinsically linked to several important properties for the molten salt, including reactivity, diffusion rates and thermophysical behavior with other species, particularly actinides, encountered during the pyroprocessing operation [5].

Metal ions in chloride salts have more complex bonding configurations than fluorides in general because of the additional oxidation states $(+7,+5,+3,+1$, and -1$)$ available for chlorine [6], leading to intriguing possible structural properties and coordination environments. The metal complex in chloride salts may have a significant impact on the salts properties that are important for their engineering applications. The most pertinent information concerning the coordination behavior of actinides in molten salts can be obtained using electronic absorption spectroscopy $[7,8]$. For investigation of the structural symmetry and chemical constitution of molten salts for application to electrorefining and molten salt reactor (MSR) applications, a versatile high-temperature apparatus for in situ spectroscopic studies of molten salts has been designed at the Idaho National Laboratory 
(INL), with a capability to combine several spectroscopy and electrochemical techniques simultaneously (discussed later in Section 2.1) [9].

The optical absorption spectra of transition metal ions have been analyzed experimentally in several molten halide salts [10-16]; however, the absorption spectra are not usually accurately obtained and compared using a computational framework [17-19]. Computational analysis to predict various properties of the salt systems, especially coordination and structural chemistry, is an area with widening interest for metal inclusions in various molten salt media. Molten salts pose a unique challenge computationally for density functional theory (DFT) methods, as the complexity of the system is relatively high, even for binary salt compositions, such as $\mathrm{LiCl}-\mathrm{KCl}$. This complexity hampers more resource-intensive methods and requires novel adaptation of existing techniques to provide meaningful results. It is relatively well known that excitonic effects play a significant role in optical spectra calculations [20-22]. However, performing Bethe-Salpeter equation calculations (which explicitly treat exitonic effects through a quasiparticle approximation using either the Green-Coulomb approximation (GW) method or the density functional perturbation theory (DFPT) method) [23] are extremely computationally intensive, especially for complex systems, such as a molten salt.

Here, we qualitatively predict the coordination behavior of the selected transition metal ions in the eutectic composition using computational methods combining quantum molecular dynamics (QMD) and the Heyd-Scuseria-Ernzerhof exchange-correlation functional (HSE-06) optical calculations, in order to correlate the coordination of transition metal ion and absorption spectra with the experimentally obtained data. To the best of the authors' knowledge, calculation of optical absorption spectra from transition metals in molten chloride melts using hybrid-functionals has not been investigated and reported. A detailed comparison with the experimentally obtained spectra for $3 \mathrm{~d}$ transition metal ions allowed the authors to determine the applicability of hybrid functional-based calculations, on which the optical spectra of two $4 \mathrm{~d}$ transition metal ions were computed. The challenges, strengths and shortcomings of two computational approaches, Perdew-Burke-Ernzerhoff (PBE) and the HSE-06 hybrid functional for the prediction of optical spectra and the coordination environment of metal species have been discussed. This experimentally validated computational approach can be applied to various salt systems to investigate the structure and coordination environment in an extended wavelength range and temperature regime.

\section{Materials and Methods}

\subsection{Apparatus}

All operations pertaining to the handling of the molten salt compositions and optical spectroscopy measurements were performed inside a glovebox containing an Ar atmosphere (Vacuum Technologies Inc., Gloucester, MA, USA) maintained at less than $0.1 \mathrm{ppm}$ moisture and oxygen concentrations. The optical absorption spectra were recorded using a Cary 5000 spectrophotometer, equipped with a deuterium and tungsten lamp used to cover wavelengths across the ultraviolet to near infrared region from 200 to $2300 \mathrm{~nm}$. The apparatus used for collecting the experimental data has been described in a previous publication [9]. The wavelength scale of the spectrophotometer in the range of 880 to $280 \mathrm{~nm}$ was verified and calibrated using a National Institute of Standards and Technology certified reference material consisting of an aqueous solution prepared from didymium perchlorate, which is permanently inside a high quality far ultraviolet (UV) quartz cell, certificate number 72,641 .

\subsection{Experimental Methods}

Anhydrous $\mathrm{LiCl}-\mathrm{KCl}$ eutectic, $\mathrm{NiCl}_{2}$ and $\mathrm{CrCl}_{3}$ of $99.99 \%$ purity (trace metals basis) were procured from Sigma-Aldrich (Burlington, MA, USA) and were used as received (no further salt purification was performed). The quartz cuvettes were rinsed with acetone and isopropanol, then dried under an evacuated Ar atmosphere at $175^{\circ} \mathrm{C}$ overnight prior to use. Custom designed quartz lids were used to minimize salt vaporization at elevated 
temperatures and were subjected to the same cleaning procedure as the cuvettes. Samples were prepared by introducing the desired masses of salts in the cleaned and dried cuvettes at room temperature, prior to loading into the furnace. Sample weights were recorded to $0.1 \mathrm{mg}$ precision using a Mettler Toledo balance. Due to the differences in the molar absorptivity of $\mathrm{NiCl}$ and $\mathrm{CrCl}_{3}$, the concentrations were adjusted to provide the highest signal to noise ratio possible without overwhelming the detection limits of the spectrophotometer. The total salt mass for each sample was approximately $5 \mathrm{~g}$. The $\mathrm{NiCl}_{2}$ and $\mathrm{CrCl}_{3}$ were loaded in $0.1 \mathrm{wt} \%$ concentrations in the $\mathrm{LiCl}-\mathrm{KCl}$ eutectic, respectively. A sample of blank $\mathrm{LiCl}-\mathrm{KCl}$ eutectic salt was used to record the baseline spectra prior to recording spectra of each species at each temperature point. The design of the furnace allowed the parallel run for all the samples at each temperature setpoint. The transition metal chlorides were allowed to dissolve in $\mathrm{LiCl}-\mathrm{KCl}$ melt at $600{ }^{\circ} \mathrm{C}$ to ensure complete/maximum dissolution; spectra were then recorded on the cool down from $600{ }^{\circ} \mathrm{C}$ to $400{ }^{\circ} \mathrm{C}$ at intervals of $50{ }^{\circ} \mathrm{C}$. At each temperature set point, the furnace was allowed to equilibrate for 30 min prior to recording the baseline and subsequent sample spectra. These samples are shown in their molten form in Figure 1.

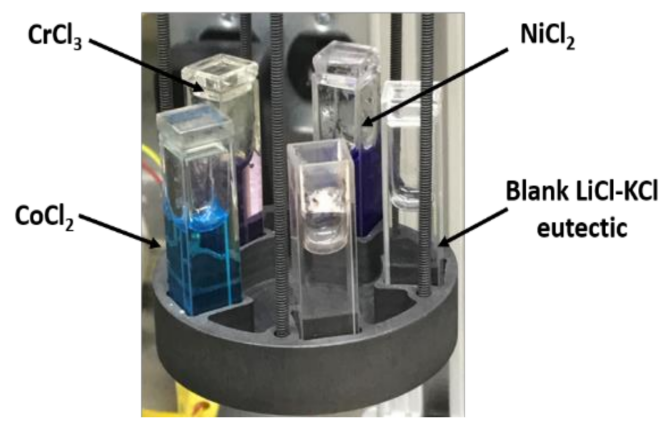

Figure 1. Molten $\mathrm{LiCl}-\mathrm{KCl}$ eutectic salt solution immediately after withdrawal from the heating furnace at $500{ }^{\circ} \mathrm{C}$ containing $\mathrm{NiCl}_{2}$ (purple color) and $\mathrm{CrCl}_{3}$ (pinkish purple) in quartz cuvette, along with the blank $\mathrm{LiCl}-\mathrm{KCl}$ eutectic (clear). Solution containing $\mathrm{CoCl}_{2}$ is shown for color comparison (blue).

\subsection{Computational Methods}

All calculations in this work were performed using the Vienna Ab-initio Simulation Package (VASP) code [24-27]. A supercell was devised to represent the LiCl-KCl eutectic composition $(59.2 \% \mathrm{~mol} \mathrm{LiCl})$ comprised of $19 \mathrm{Li}$ atoms, $32 \mathrm{Cl}$ atoms, and $13 \mathrm{~K}$ atoms, which is shown in Figure S1 of the Supplemental Materials (SM). The initial structure was constructed based on experimental density [14] and the atoms are randomly distributed in the supercell. Then, quantum molecular dynamics (QMD) was performed using the PBE functional $[28,29]$ to optimize the structure through a rapid quenching simulation beginning with heating to $\mathrm{T}=2000 \mathrm{~K}$ (a temperature well above the melting point) for $5 \mathrm{ps}$ to ensure the constituent atoms in the simulation reached sufficient equilibrium for liquid behavior prior to further refinement. Each system was then "quenched" in the simulation from $2000 \mathrm{~K}$ to the target temperature rapidly over a period of an additional $5 \mathrm{ps}$. Finally, each system was equilibrated for a longer period of time (with a minimum of $10 \mathrm{ps)} \mathrm{at}$ the fixed target temperature. Equilibration was performed continuously with adjusting volume in all cases until the pressure of the system converged to near zero and the energy of the system was deemed to be converged. Figure S1 shows the energy convergence for the $\mathrm{Ni}^{2+}$ in the simulated $\mathrm{LiCl}-\mathrm{KCl}$ eutectic as an example. Figure $\mathrm{S} 2$ shows the optimized $\mathrm{LiCl}-\mathrm{KCl}$ eutectic system after equilibration, as described above.

In order to simulate the inclusion of the three transition metals targeted in this study, a corresponding quantity of $\mathrm{K}$ and $\mathrm{Li}$ atoms were removed and replaced with the transition metal cation prior to the high-temperature simulation in order to preserve the nearestpossible composition to the eutectic. These systems were quenched to temperatures of 
$400{ }^{\circ} \mathrm{C}, 500^{\circ} \mathrm{C}$ and $600^{\circ} \mathrm{C}$ then equilibrated at the target temperature in order to allow direct comparison with the experimentally obtained results for each transition metal-molten salt system. Additionally, in order for the proper bonding behavior to be exhibited in the QMD, each calculation containing a metal cation was treated with spin polarization specified to ensure accurate results. $\mathrm{Ni}$ and $\mathrm{Cr}$ were treated with the per-atom magnetic moment of 0.6 and $0.7 \mu \mathrm{B}$, respectively. The energy cutoff for the wavefunction expansion was specified to be $400 \mathrm{eV}$. The self-consistent field (SCF) convergence criteria were designated as $1 \times 10^{-5} \mathrm{eV}$ with a maximum number of 200 cycles. Normal precision was used for computational simplicity due to the complexity of the simulated system. Fermi smearing was applied to all QMD calculations with a smearing width of $0.172 \mathrm{eV}$. All visualization of QMD results, including Figure 2 and Figure S2 were performed using the VESTA software (Koichi Momma, Ibaraki, Japan) [30].
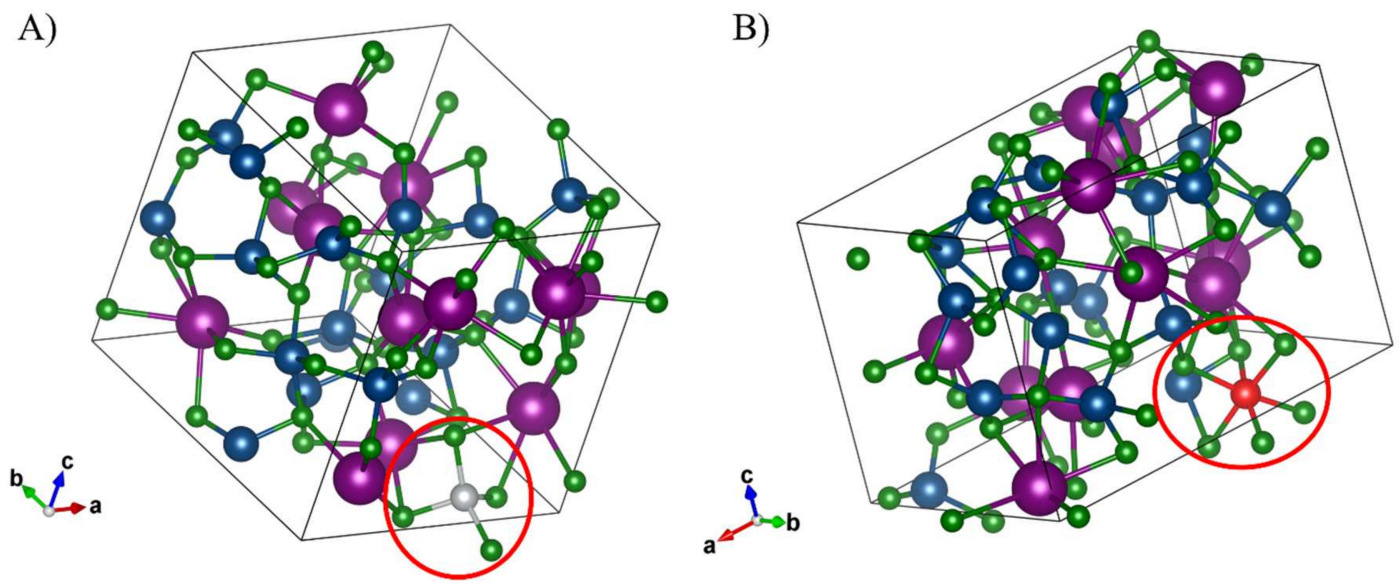

Figure 2. The transition metal chlorocomplex is circled in red, with the $3 \mathrm{~d}$ cation shown in grey $(\mathrm{Ni})$ or red $(\mathrm{Cr})$ and $\mathrm{Li} / \mathrm{Cl} / \mathrm{K}$ shown in blue, green and purple, respectively. Configuration of (A) coordination of $\mathrm{NiCl}_{4}{ }^{2-}$ chlorocomplex and (B) $\mathrm{CrCl}_{6}{ }^{3-}$ chlorocomplex, in $\mathrm{LiCl}-\mathrm{KCl}$ eutectic composition at $400{ }^{\circ} \mathrm{C}$. The chlorocomplex is circled in red in each case for clarity.

Optical spectrum calculations were performed using the VASP calculation method explained by Gadjos et al. [31], in which a Kubo-Greenwood style linear response was calculated for both the real and imaginary portions of the dielectric function, with the imaginary portion being used to calculate optical properties, including absorption. The imaginary portion is calculated according to the following equation:

$$
\varepsilon \frac{i m}{\alpha \beta}(\omega)=\frac{4 \pi^{2} e^{2}}{\Omega} \lim _{q \rightarrow 0} \frac{1}{q^{2}} \sum c, v, k 2 W_{k} \delta\left(\epsilon_{c k}-\epsilon_{v k}-\omega\right) \times\left\langle U_{c k+e_{\alpha} q} \mid U_{v k}\right\rangle\left\langle U_{c k+e_{\beta} q} \mid U_{v k}\right\rangle
$$

The details of the derivation of the absorption spectrum from the real and imaginary dielectric tensor components are explained in detail in previous work [31]. In Equation (1), $c$ represents the conduction band states specified in initial input for the calculation, while $v$ indicates the valence band states for the system obtained from the band structure calculation and $k$ represents the $k$-point for this specific calculation. This means that, as the imaginary dielectric function is evaluated at each $k$-point for conduction and valence bands $c$ and $v$, increasing any of these three values by evaluating a denser $k$-point mesh or specifying more accurate valence and conduction band settings (approaching the total number of bands, NBANDS) drastically increases the computational requirements of the calculation. In this calculation method the NBANDS value was increased to three times the total valence electrons in the simulated system in order to allow for a better calculation of the dielectric tensor, while the values of $c$ and $v$ were kept to the minimal values in order to lower the computational requirements. 
Optical calculations were carried out according to a two-part schema, in which the ground state band structure and wave functions for the target system were calculated in a single calculation, followed by an optical calculation to derive the frequency-dependent dielectric tensor, as described previously. The VASPKIT (Wang et. al) post-processing software [32] was used to derive the absorption spectra for each species.

For the optical calculations, both the PBE functional and the HSE-06 functional were evaluated. The PBE functional was found to be unsatisfactory for use due to inaccurate band structure calculations stemming from underestimation of the band gap for complex systems. The hybrid HSE-06 [33] functional, using a 25/75 mix of Hartree-Fock and DFT calculations, was found to result in a significantly more accurate absorption spectrum for each species relative to the experimentally observed spectrum than the PBE functional, due to its more accurate band structure calculation for each system leading to a more-accurate dielectric tensor obtained from the resulting wave functions. Similarly, the k-points mesh was chosen to be the relatively simple gamma-centered Monkhorst-Pack $2 \times 2 \times 2$ configuration in order to minimize computational requirements due to the complexity of the unit cell, as finer $k$-point meshes were found to be prohibitively computationally expensive. Gaussian smearing was used in all calculations, and the complex shift $\eta$ was approximated to be $0.100 \mathrm{eV}$.

Due to the inherent fluctuations of the molecular dynamics approach and to improve the quality of the resultant band structure for dielectric tensor computation, a trajectory average was obtained by averaging the individual positions of each atom from the QMD across a designated "timespan" of timesteps in order to obtain an averaged overall structure for the eutectic salt composition in each case. Averages obtained from individual positions across timestep spans of $>0.6$ ps were found to exacerbate bond angle issues with the transition metal chlorocomplexes, while $<0.3$ ps averages were found to vary significantly from each other over the course of the MD. As such, all optical results were obtained from averages of the individual positions from 0.6 ps (600 MD steps in VASP using the default timestep of $1 \mathrm{fs}$ ) of the QMD.

\section{Results and Discussions}

The $\mathrm{LiCl}-\mathrm{KCl}$ solution containing $\mathrm{NiCl}_{2}$ and $\mathrm{CrCl}_{3}$ in a molten state are shown in Figure 1. The $\mathrm{NiCl}_{2 \mathrm{a}}$ solution is characterized as a vibrant purple color in the chloride melts, while $\mathrm{CrCl}_{3}$ obtains a pinkish translucent color in the melt. The blank $\mathrm{LiCl}-\mathrm{KCl}$ eutectic is transparent in molten state. The color of each melt remained unchanged through the temperature range investigated.

The $\mathrm{d}-\mathrm{d}$ transitions, and hence the geometrical arrangement of the central $3 \mathrm{~d}$ metal ion, are easily influenced by the fields of the nearby ions and molecular anions, as the $3 \mathrm{~d}$ shell electrons are only lightly protected by the outer electrons. Due to the partially filled $\mathrm{d}$ shells, the spectrum of transition-metal ions is characteristic of broad, weak bands in the near-IR and visible spectral regions and high intensity UV bands. The bands in the near-IR and visible range are generated by $\mathrm{d}$ - $\mathrm{d}$ transitions, or in other words, transitions within specific energy levels of $\mathrm{d}^{\mathrm{n}}$ electronic configurations. The strong bands in the ultraviolet are known as charge-transfer transitions and occur due to transfer of $p$ electrons from a ligand anion (a halide ion in this case) into an enlarged orbital that is confined by nearby cations.

The coordination of $\mathrm{Ni}^{2+}$ and $\mathrm{Cr}^{3+}$ chlorocomplexes in the $\mathrm{LiCl}-\mathrm{KCl}$ eutectic solution are shown in Figure 2A,B, respectively. $\mathrm{Ni}^{2+}$ is found to adopt a tetrahedral coordination, while $\mathrm{Cr}^{3+}$ is found to adopt an octahedral coordination. The optical absorption spectra of the selected species in the $\mathrm{LiCl}-\mathrm{KCl}$ eutectic media are shown in Figures 3-5. For $\mathrm{Ni}^{2+}$ and $\mathrm{Cr}^{3+}$, the important charge transfer band found in the lower wavelength range was observed to shift to lower energies with increased temperature. This charge transfer band shift is similar to what has been observed for $\mathrm{Ni}^{2+}$ and $\mathrm{Co}^{2+}$ in $\mathrm{ZnCl}_{2}[12,34]$ and is attributed to the increased thermal energy of the system leading to a corresponding lowered energy requirement for the resulting charge transfer between $\mathrm{Cl}^{-}$and the relevant cation [35]. 


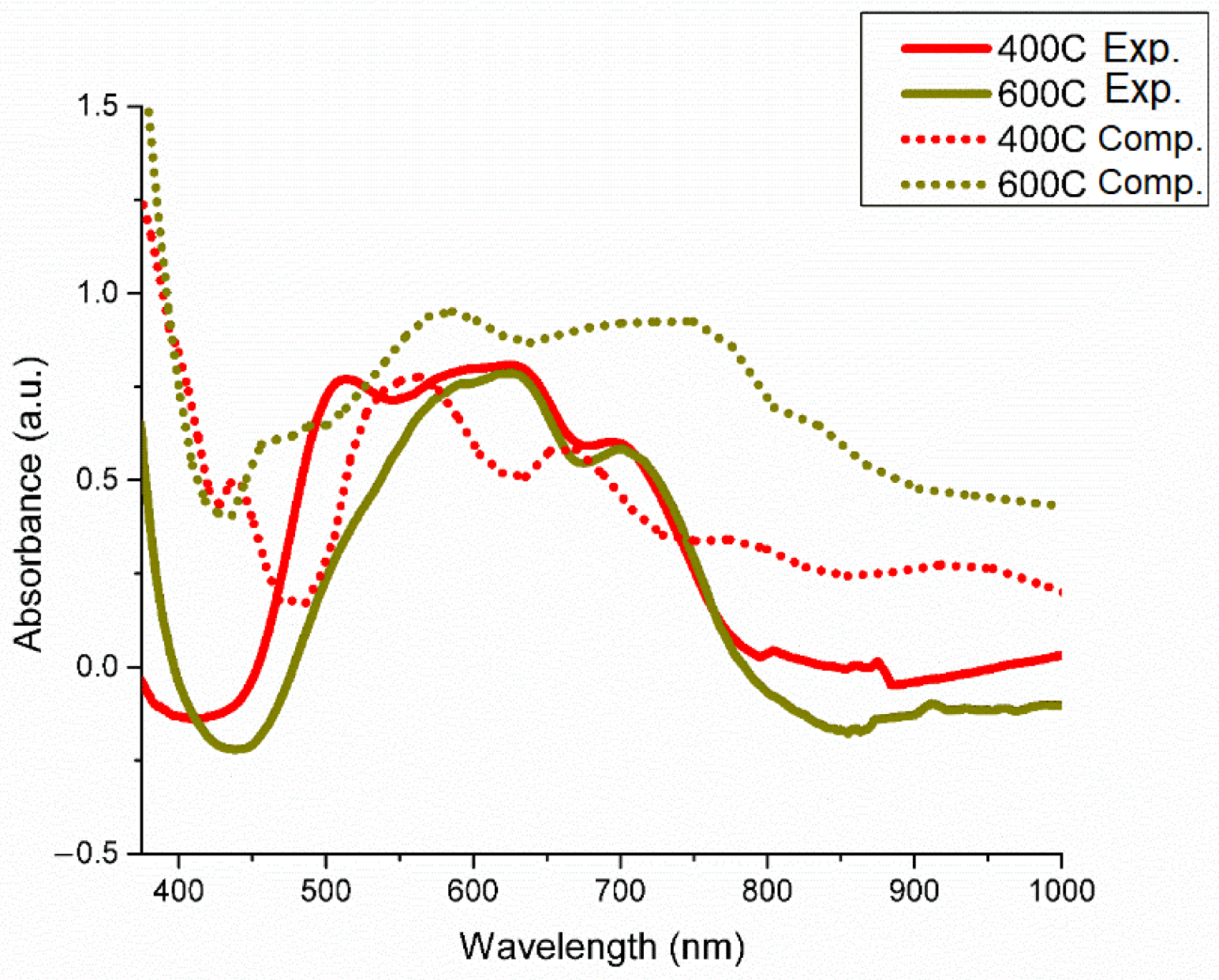

Figure 3. Experimental and computationally obtained optical absorption spectra for $\mathrm{Ni}^{2+}$ in the $\mathrm{LiCl}-\mathrm{KCl}$ eutectic system as a function of temperature. Computational spectra at $400{ }^{\circ} \mathrm{C}$ and $600{ }^{\circ} \mathrm{C}$ demonstrate moderate correlation with experimental results, with expected blue shift in peak location.

The calculated optical absorption spectra obtained using the HSE-06 hybrid functional is likewise presented in Figures 3-5. Exitonic effects, not explicitly treated in hybrid functional calculations, and their impact on the calculated optical spectra are visible in all cases, leading to blue shift of signature features for each element with varying degrees of severity along with distortion of the peak profiles. The configuration of each species obtained from QMD is shown in Figure 2, with each agreeing with the experimental results obtained here and in previous works [14]. For $\mathrm{Ni}^{2+}$ in the $\mathrm{LiCl}-\mathrm{KCl}$ eutectic system, temperature effects were also investigated computationally to demonstrate the relation between the strengthened tetrahedral coordination at higher temperatures theorized to explain the difference in experimental spectra across various temperatures.

The optical absorption spectrum of $\mathrm{NiCl}_{2}$ dissolved in $\mathrm{LiCl}-\mathrm{KCl}$ has been experimentally studied as a function of temperature by Boston and Smith [29], Jorgensen [16] and Gruen et al. [14] The changes in the features of the spectrum with increasing temperature have been previously assigned to the changes in the population ratio of the tetrahedral and octahedral nickel chlorocomplexes in the system. An alternative explanation for this behavior was proposed by Sundheim and Harrington [34], and was based on theoretical reasons that the spectrum primarily corresponds to the tetracoordinated nickel ions with bond angle distortion from optimal tetrahedral symmetry that increases as temperature decreases towards the melting point of the system. Based on a recent study by one of the coauthors on $\mathrm{Ni}^{2+}$ in $\mathrm{ZnCl}_{2}$ media, $\mathrm{Ni}^{2+}$ seems to adopt 'distorted' tetrahedral geometry, which is validated by both optical spectroscopy and AIMD simulations [34]. In this case 
as well, distorted $\mathrm{T}_{\mathrm{d}}$ geometry is observed for $\mathrm{Ni}^{2+}$ at low temperatures. However, with increase in temperature above $450{ }^{\circ} \mathrm{C}$, the complex transitions into a pure $\mathrm{T}_{\mathrm{d}}$ environment. Such change in symmetry can be viewed as the stark decrease in the intensity of the feature around $500 \mathrm{~nm}$ with temperature.

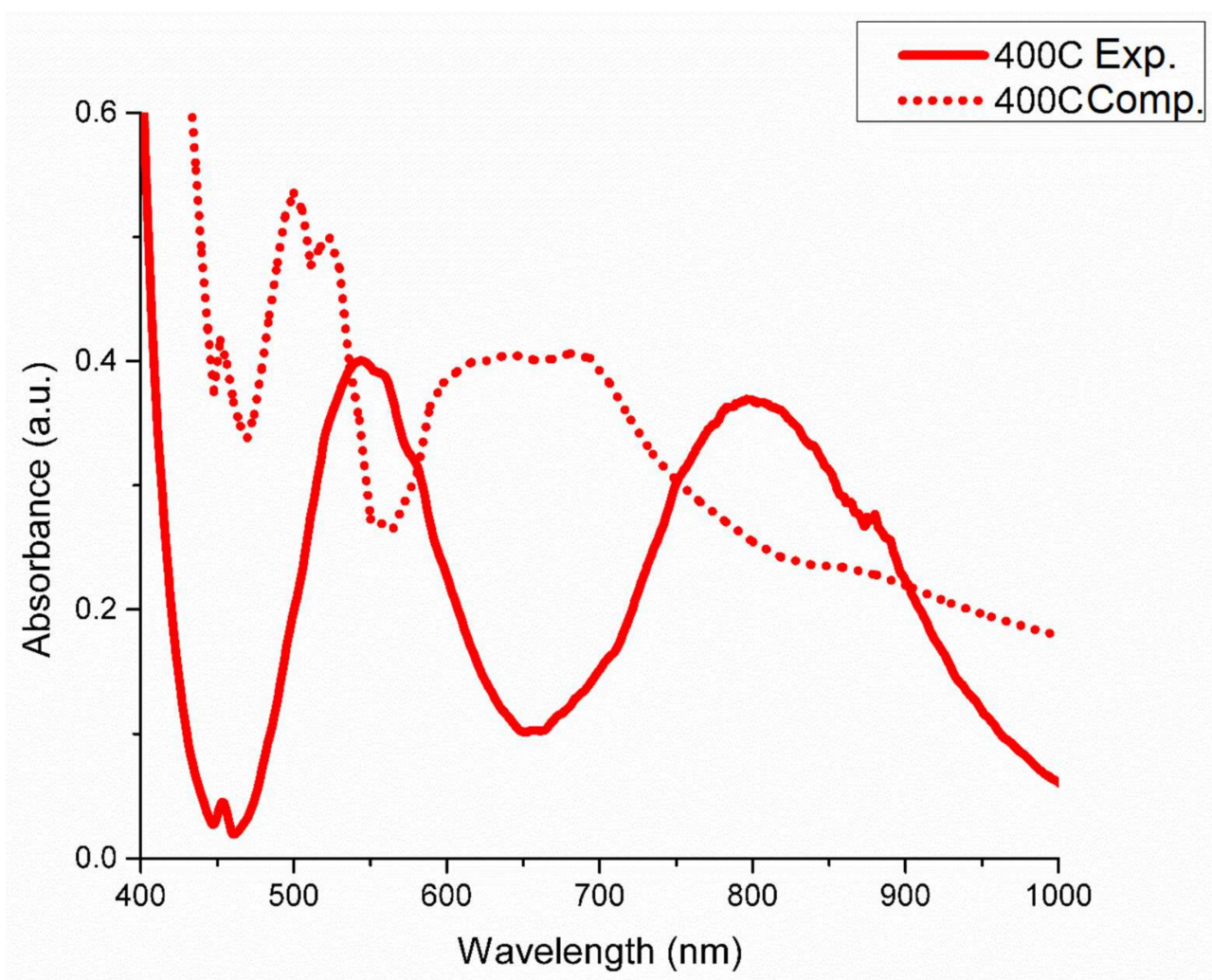

Figure 4. Experimental and computational optical absorption spectra of $\mathrm{Cr}^{3+}$ in $\mathrm{LiCl}-\mathrm{KCl}$ eutectic melt at $400{ }^{\circ} \mathrm{C}$, with the solid line representing the experimentally obtained spectrum and the dotted line representing the computational absorbed spectrum using the HSE-06 functional. Significant blue shift is evident for both peaks in the computational spectrum.

The differences in the distorted tetrahedral and ordinary tetrahedral coordination of $\mathrm{Ni}^{2+}$ in the form of the $\mathrm{NiCl}_{4}{ }^{2-}$ chlorocomplex (shown in Figure 2) are visible in our computationally obtained spectra, shown in Figure 3 and Figure S3 of the SI. Our calculations indicate the $400{ }^{\circ} \mathrm{C}$ HSE-06 spectra (dotted red line) to contain the distorted three-peak key feature shown in Figure 3, which is notably diminished in the $600{ }^{\circ} \mathrm{C}$ feature shown (dotted gold line). As with the $\mathrm{Cr}^{3+}$ system, a degree of blue shift tied to the exitonic effects in the system is present in the spectrum, although to a more muted degree.

The orientation of the $\mathrm{NiCl}_{4}{ }^{2-}$ bond angles likewise change between the two target temperatures in the QMD, with the average $\mathrm{Ni}-\mathrm{Cl}$ bond angle after equilibration at 400 ${ }^{\circ} \mathrm{C}$ found to be $112.4^{\circ}$ and at $600{ }^{\circ} \mathrm{C}$ the average bond angle was found to be $110.1^{\circ}$, significantly closer to the ideal $109.5^{\circ}$ for a tetrahedral complex. These results indicate a transition towards a pure tetrahedral configuration at increased temperatures, although some distortion in bond angles remains due to the forces inherent in the QMD. This supports the temperature-dependent distorted tetrahedral symmetry concept, previously proposed by Sundheim and Harrington [34]. 
A) $\mathrm{Mo}^{3+}$ in $\mathrm{LiCl}-\mathrm{KCl}$

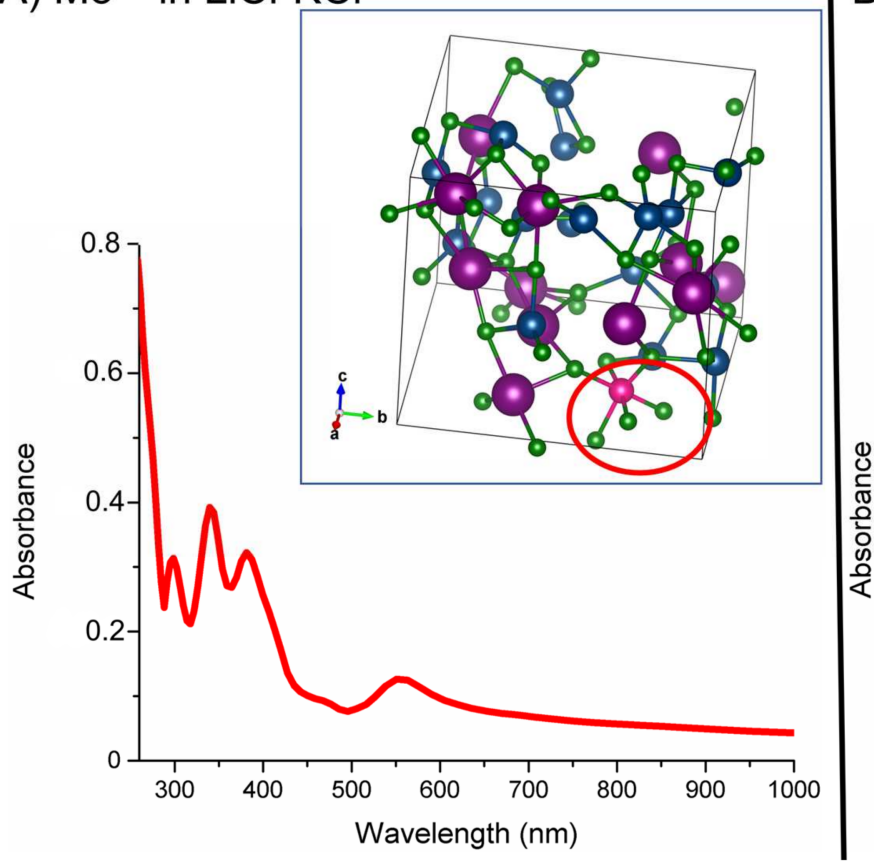

B) $\mathrm{Nb}^{3+}$ in $\mathrm{LiCl}-\mathrm{KCl}$

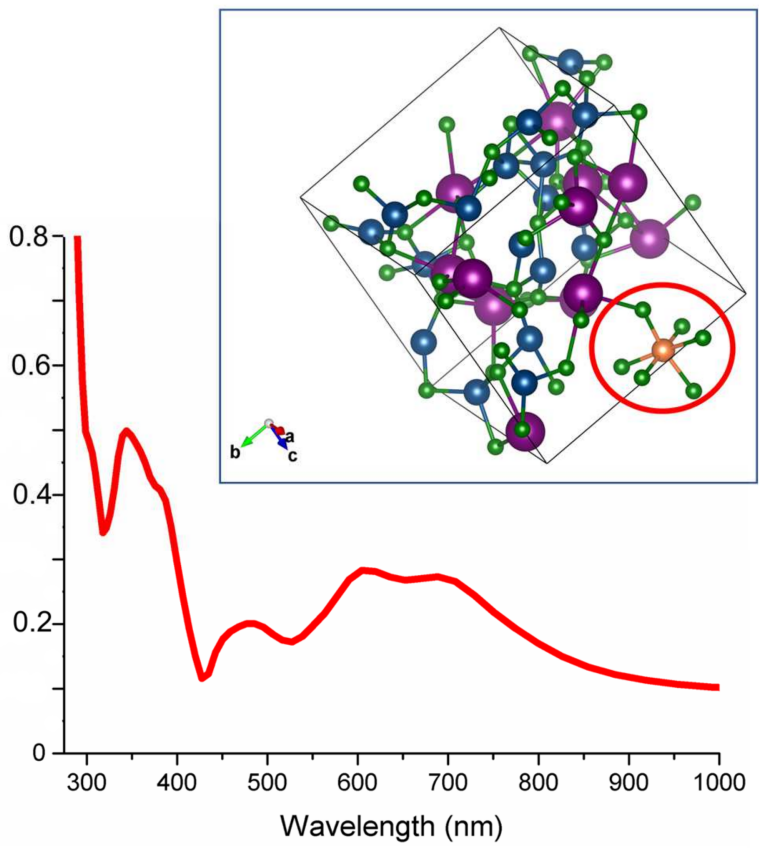

Figure 5. The transition metal chlorocomplex is circled in red, with the $4 \mathrm{~d}$ cation shown as a pink $(\mathrm{Mo})$ or orange $(\mathrm{Nb})$ sphere, and $\mathrm{Li} / \mathrm{Cl} / \mathrm{K}$ is shown as blue, green and purple spheres, respectively. (A) $\mathrm{MoCl}_{6}{ }^{3-}$ chlorocomplex in $\mathrm{LiCl}-\mathrm{KCl}$ eutectic at $400{ }^{\circ} \mathrm{C}$. (B) $\mathrm{NbCl}_{6}{ }^{3-}$ chlorocomplex in $\mathrm{LiCl}-\mathrm{KCl}$ eutectic at $400{ }^{\circ} \mathrm{C}$. (A) Computationally obtained optical absorption spectra for $\mathrm{Mo}^{3+}$ in $\mathrm{LiCl}-\mathrm{KCl}$ eutectic composition at $400{ }^{\circ} \mathrm{C}$. (B) Computationally obtained optical absorption spectra for $\mathrm{Nb}^{3+}$ in $\mathrm{LiCl}-\mathrm{KCl}$ eutectic composition at $400{ }^{\circ} \mathrm{C}$.

The optical absorption spectrum of trivalent $\mathrm{Cr}$ has been previously studied by Harrington and Sundheim [36], and also by Gruen and McBeth [14] in the LiCl-KCl eutectic. The spectrum of trivalent $\mathrm{Cr}$ in $\mathrm{LiCl} \cdot \mathrm{KCl}$ was found by both groups to correspond to octahedral $\mathrm{CrCl}_{6}{ }^{3-}$ ions. The spectrum contains two bands in the visible region, assigned as follows: $800 \mathrm{~nm}$ to ${ }^{4} \mathrm{~A}_{2 \mathrm{~g}}(\mathrm{~F}) \rightarrow{ }^{4} \mathrm{~T}_{2 \mathrm{~g}}(\mathrm{~F})$, and $540 \mathrm{~nm}$ to ${ }^{4} \mathrm{~A}_{2 \mathrm{~g}}(\mathrm{~F}) \rightarrow{ }^{4} \mathrm{~T}_{1 \mathrm{~g}}(\mathrm{~F})$. There are no prominent features in the near-IR range. The spectral features and thus the geometry for the $\mathrm{Cr}^{3+}$ chlorocomplex remains consistent over the temperature range investigated. This is consistent with QMD results, shown in Figure 2A, where a strong octahedral $\mathrm{CrCl}_{6}{ }^{3-}$ coordination is evident at all investigated temperatures. The calculated spectrum at $400{ }^{\circ} \mathrm{C}$ is shown in Figure 4 with a dotted line and exhibits some peculiarities compared to the experimentally obtained results, likely due to bond-angle distortion from the MD and the untreated exitonic effects inherent to our calculation method. Importantly, the characteristic dual transitions corresponding to the $540 \mathrm{~nm}$ and $800 \mathrm{~nm}$ are present, although $540 \mathrm{~nm}$ peak is blue-shifted roughly $40 \mathrm{~nm}$ and the $800 \mathrm{~nm}$ peak is blue-shifted significantly to $\sim 700 \mathrm{~nm}$ in the computational spectrum. The spectra for $\mathrm{Cr}^{3+}$ in $\mathrm{LiCl}-\mathrm{KCl}$ eutectic were found to not be significantly temperature dependent, unlike $\mathrm{Ni}^{2+}$, in either computational or experimental analysis.

To further test our computational approach, we also analyzed the $4 \mathrm{~d}$ transition metals, $\mathrm{Mo}$ and $\mathrm{Nb}$, in the $\mathrm{LiCl}-\mathrm{KCl}$ eutectic composition previously described. In Figure 5, the computed coordination of $\mathrm{Mo}^{3+}$ and $\mathrm{Nb}^{3+}$ at $400{ }^{\circ} \mathrm{C}$ is shown along with the computationally obtained optical absorbance using the HSE-06 hybrid functional. We find that both $\mathrm{Mo}$ and $\mathrm{Nb}$ obtain an octahedral structure in the eutectic melt $\left(\mathrm{MoCl}_{6}{ }^{3-}\right.$ and $\left.\mathrm{NbCl}_{6}{ }^{3-}\right)$ at $400{ }^{\circ} \mathrm{C}$. This observation is in good agreement with prior experimental research on the two species in the $\mathrm{LiCl}-\mathrm{KCl}$ eutectic melt $[37,38]$.

Mo can potentially adopt several coordination states due to disproportion in the $\mathrm{LiCl}-$ $\mathrm{KCl}$ eutectic, depending on a variety of conditions found under prior electrochemistry 
experiments, including the electrochemically important $\mathrm{MoCl}_{6}{ }^{3-}$ and byproduct $\mathrm{MoCl}_{5}$ and $\mathrm{MoCl}_{4}{ }^{2-}$ chlorocomplexes, both of which are believed to be highly volatile [37]. Therefore, our efforts were focused on the $\mathrm{Mo}^{3+}$ coordination to obtain the coordination in $\mathrm{LiCl}-$ $\mathrm{KCl}$. The coordination of $\mathrm{Mo}^{3+}$ in the form of $\mathrm{MoCl}_{6}{ }^{3-}$ is shown in Figure $5 \mathrm{~A}$; the Mo cation forms an octahedral chlorocomplex with somewhat distorted bond angles from the preferred $90^{\circ}$, likely due to the inherent forces in the MD technique applied. The octahedral coordination is visible in the optical absorption spectra shown in Figure 5A, where a double peak feature is found reminiscent of the octahedral spectra obtained for $\mathrm{Cr}^{3+}$ in Figure 4 . The experimental results reported by Gabriel et al. lacked a conclusive coordination state for $\mathrm{Mo}$ in $\mathrm{LiCl}-\mathrm{KCl}$ primarily due to its high volatility. Our computational results signify the formation of a distorted octahedral complex $\mathrm{MoCl}_{6}{ }^{3-}$ for $\mathrm{Mo}^{3+}$ at $400{ }^{\circ} \mathrm{C}$.

Likewise, $\mathrm{Nb}$ has also been found to adopt multiple coordination states in $\mathrm{LiCl}-$ $\mathrm{KCl}$ [38], and we have chosen to focus on the predominant $\mathrm{Nb}^{3+}$ coordination for our computational results. The octahedral $\mathrm{NbCl}_{6}{ }^{3-}$ chlorocomplex forms in the simulated eutectic, as shown in Figure 5B; the average bond angle for the chlorocomplex was found to be $92.1^{\circ}$, which is much closer to the ideal $90^{\circ}$ than our results for the similarly behaving $\mathrm{MoCl}_{6}{ }^{3-}$. This stronger octahedral geometry for the chlorocomplex is evident in the absorption spectra shown in Figure 5B, where the two-peak feature is more readily recognizable as an octahedral spectra. We find reasonable qualitative agreement with the $\mathrm{LiCl}-\mathrm{KCl}$ eutectic results reported by Brevnova et al., who similarly reported an octahedral spectra for $\mathrm{NbCl}_{6}{ }^{3-}$ with absorption peaks at 480 and $650-700 \mathrm{~nm}$ that correspond to the ${ }^{3} \mathrm{~T}_{1 \mathrm{~g}} \rightarrow{ }^{3} \mathrm{~T}_{1 \mathrm{~g}}(\mathrm{P})$ and ${ }^{3} \mathrm{~T}_{1 \mathrm{~g}} \rightarrow{ }^{3} \mathrm{~T}_{2 \mathrm{~g}}$ transitions. We similarly find a broad feature in the $600-700 \mathrm{~nm}$ range paired with a narrower feature at around $475 \mathrm{~nm}$, shown in Figure 5B above.

\section{Conclusions}

We report the experimental and computational optical absorption spectra (using the HSE-06 hybrid functional) for four transition metal chlorocomplexes important to pyroprocessing and corrosion processes in the $\mathrm{LiCl}-\mathrm{KCl}$ binary eutectic salt composition, including those formed by $\mathrm{Ni}^{2+}, \mathrm{Cr}^{3+}, \mathrm{Mo}^{3+}$ and $\mathrm{Nb}^{3+}$ as well as their predicted coordinations using QMD. We find from the QMD that $\mathrm{Ni}^{2+}$ forms the tetrahedral $\mathrm{NiCl}_{4}{ }^{2-}$ chlorocomplex, while $\mathrm{Cr}^{3+}, \mathrm{Mo}^{3+}$ and $\mathrm{Nb}^{3+}$ form the octahedral $\mathrm{CrCl}_{6}{ }^{3-}, \mathrm{MoCl}_{6}{ }^{3-}$ and $\mathrm{NbCl}_{6}{ }^{3-}$ chlorocomplexes, respectively. The results obtained agree with previous experimental conclusions and are supported by the optical spectra obtained both experimentally and computationally in this work.

Experimental spectra for $\mathrm{Ni}^{2+}$ indicates a shift from distorted tetrahedral to distinct tetrahedral as the temperature increases, with distorted tetrahedral three-peak spectra found for temperatures below $450{ }^{\circ} \mathrm{C}$ and two-peak spectra found at higher temperatures; our QMD results support this, with the tetrahedral $\mathrm{NiCl}_{4}{ }^{2-}$ chlorocomplex forming at all temperatures and with a decrease of average bond angle towards the $109.5^{\circ}$ tetrahedral ideal found in optimized position-averaged systems at higher temperatures. We likewise found more distinct two-peak spectra in computational optical absorption spectra at elevated temperatures. Computational optical absorption spectra for $\mathrm{Cr}^{3+}, \mathrm{Mo}^{3+}$ and $\mathrm{Nb}^{3+}$ all featured separated two-peak spectra indicative of octahedral coordinations, with $\mathrm{Cr}^{3+}$ showing blue shift compared to experimental results for both characteristic features. All computational optical spectra were found to have deviation from the experimental spectra, likely due to the untreated excitonic effects that require a separate quasiparticle calculation evaluated, using the Beth-Salpeter equation (BSE) method. The full BSE treatment is, however, difficult to perform for a system of this complexity due to the intense computational resource requirements. A natural development of these computational optical spectroscopy methods is finding a way to minimize the resources of such a calculation to evaluate the optical spectra of chlorocomplexes more accurately in molten salts systems. 
Supplementary Materials: The following supporting information can be downloaded at: https: / / www.mdpi.com/article/10.3390/ma15041478/s1, The SM file contains Figure S1-Energy convergence of $\mathrm{Ni}^{2+}$ in $\mathrm{LiCl}-\mathrm{KCl}$ eutectic at $400{ }^{\circ} \mathrm{C}$, Figure $\mathrm{S} 2-\mathrm{LiCl}-\mathrm{KCl}$ eutectic composition from molecular dynamics simulations at $400{ }^{\circ} \mathrm{C}$ and Figure $\mathrm{S} 3-\mathrm{Ni}^{2+}$ in $\mathrm{LiCl}-\mathrm{KCl}$ at $400{ }^{\circ} \mathrm{C}$, obtained experimentally and from the HSE optical calculation. The .xlsx file contains all computationally and experimentally obtained optical spectra data as well as the initial positions for all MD calculations.

Author Contributions: R.G. designed optical absorption experiments. Q.A. designed computational simulations. R.G. and W.P. performed optical absorption experiments. J.F. performed DFT calculations. All authors contributed to the writing of this manuscript. All authors have read and agreed to the published version of the manuscript.

Funding: This work was supported through the INL Laboratory Directed Research \& Development (LDRD) Program under DOE Idaho Operations Office Contract DE-AC07-05ID14517.

Data Availability Statement: The data presented in this study are available in the supplementary materials in a .xlsx file.

Conflicts of Interest: The authors declare no conflict of interest.

\section{References}

1. Volkov, S.V. Coordination Compounds in Melts. In Molten Salts: From Fundamentals to Applications; Springer: Dordrecht, The Netherlands, 2012; pp. 357-373.

2. Sridharan, K.; Allen, T.R. Corrosion in Molten Salts. In Molten Salts Chemistry; Elsevier Inc.: Amsterdam, The Netherlands, 2013; pp. 241-267.

3. Li, S.X. Anodic Process of Electrorefining Spent Nuclear Fuel in Molten LiCl-KCl-UCl3/Cd System. ECS Proc. Vol. 2002, $2002,541$. [CrossRef]

4. Zhang, J. Electrochemistry of actinides and fission products in molten salts-Data review. J. Nucl. Mater. 2014, 447, 271-284. [CrossRef]

5. Barbanel, Y.A.; Kolin, V.V.; Kotlin, V.P.; Lumpov, A.A. Coordination chemistry of actinides in molten salts. J. Radioanal. Nucl. Chem. Artic. 1990, 143, 167-179. [CrossRef]

6. Harrison, T.J.; Holcomb, D.E.; Flanagan, G.F.; Patton, B.W.; Gehin, J.C.; Howard, R.L. Fast Spectrum Molten Salt Reactor Options; ORNL: Oak Ridge, TN, USA, 2011. Available online: https:/ /info.ornl.gov/sites/publications/Files/Pub29596.pdf (accessed on 22 November 2021).

7. Fujii, T.; Nagai, T.; Sato, N.; Shirai, O.; Yamana, H. Electronic absorption spectra of lanthanides in a molten chloride: II. Absorption characteristics of neodymium(III) in various molten chlorides. J. Alloys Compd. 2005, 393, L1-L5. [CrossRef]

8. Yatsimirskii, K.B. Spectroscopic studies on coordination compounds formed in molten salts. In Non-Aqueous Solutions-5; Pergamon: Leeds, UK, 1977. [CrossRef]

9. $\quad$ Phillips, W.C.; Gakhar, R.; Horne, G.P.; Layne, B.; Iwamatsu, K.; Ramos-Ballesteros, A.; Shaltry, M.R.; LaVerne, J.A.; Pimblott, S.M.; Wishart, J.F. Design and performance of high- temperature furnace and cell holder for in situ spectroscopic, electrochemical, and radiolytic investigations of molten salts Design and performance of high-temperature furnace and cell holder for in situ spectroscopic. Rev. Sci. Instrum. 2020, 91, 083105. [CrossRef]

10. Smith, G.P.; James, D.W.; Boston, C.R. Optical Spectra of $\mathrm{Tl}^{+}, \mathrm{Pb}^{2+}$, and $\mathrm{Bi}^{3+}$ in the Molten Lithium Chloride-Potassium Chloride Eutectic. J. Chem. Phys. 1965, 42, 2249-2250. [CrossRef]

11. Brynestad, J.; Boston, C.R.; Smith, G.P. Electronic spectra and coordination of nickel centers in liquid lithium chloride-potassium chloride mixtures. J. Chem. Phys. 1967, 47, 3179-3189. [CrossRef]

12. Smith, W.E.; Brynestad, J.; Smith, G.P.; Smith, W.E.; Brynestad, O.; Smitht, G.P. Spectroscopic Behavior and Coordination of Nickel(II) in Liquid Mixtures of Zinc and Cesium Chlorides Spectroscopic Behavior and Coordination of Nickel(II) in Liquid Mixtures of Zinc and Cesium Chlorides. J. Chem. Phys. 1970, 52, 3890. [CrossRef]

13. Gruen, D.M.; Division, C. Octahedral and tetrahedral co-ordination states of cobalt (II) in molten zinc chloride-aluminium chloride mixtures. J. Inorg. Nucl. Chem. 1967, 29, 2243-2247.

14. Gruen, D.M.; McBeth, R.L. The coordination chemistry of 3d transition metal ions in fused salt solutions. Pure Appl. Chem. 1963, 6, 23-48. [CrossRef]

15. Brookes, H.C.; Flengas, S.N. Spectroscopy of chromium compounds in molten salts. Can. J. Chem. 1970, 48, 55-58. [CrossRef]

16. Jørgensen, C.K. Ligand field bands of four-coordinated paramagnetic nickel(ii) complexes. Mol. Phys. 1958, 1, 410-412. [CrossRef]

17. Cho, Y.H.; Kim, T.J.; Park, Y.J.; Im, H.J.; Song, K. Electronic absorption spectra of Sm(II) and Yb(II) ions in a LiCl-KCl eutectic melt at $450{ }^{\circ}$ C. J. Lumin. 2010, 130, 280-282. [CrossRef]

18. Banks, C.V.; Heusinkveld, M.R.; O'laughlin, J.W. Absorption Spectra of the Lanthanides in Fused Lithium Chloride-Potassium Chloride Eutectic. Anal. Chem. 1961, 33, 1235-1240. [CrossRef] 
19. Kim, B.Y.; Yun, J.I. Optical absorption and fluorescence properties of trivalent lanthanide chlorides in high temperature molten LiCl-KCl eutectic. J. Lumin. 2016, 178, 331-339. [CrossRef]

20. Yang, L.; Cohen, M.L.; Louie, S.G. Excitonic effects in the optical spectra of graphene nanoribbons. Nano Lett. 2007, 7, 3112-3115. [CrossRef]

21. Marini, A.; Del Sole, R. Dynamical excitonic effects in metals and semiconductors. Phys. Rev. Lett. 2003, 91, 176402. [CrossRef]

22. Kunz, B.; Flynn, C.P. Excitonic effects in the interband spectra of metals. Phys. Rev. Lett. 1983, 50, 1524-1527. [CrossRef]

23. Wang, H. Excitonic effects and optical spectra of graphene nanoflakes. J. Appl. Phys. 2017, 122, 084301. [CrossRef]

24. Kresse, G. Ab initio molecular dynamics for liquid metals. J. Non. Cryst. Solids 1995, 192-193, 222-229. [CrossRef]

25. Kresse, G.; Hafner, J. Ab initio molecular-dynamics simulation of the liquid-metalamorphous- semiconductor transition in germanium. Phys. Rev. B 1994, 49, 14251-14269. [CrossRef]

26. Kresse, G.; Furthmüller, J. Efficiency of ab-initio total energy calculations for metals and semiconductors using a plane-wave basis set. Comput. Mater. Sci. 1996, 6, 15-50. [CrossRef]

27. Kresse, G.; Furthmüller, J. Efficient iterative schemes for ab initio total-energy calculations using a plane-wave basis set. Phys. Rev. B-Condens. Matter Mater. Phys. 1996, 54, 11169-11186. [CrossRef]

28. Perdew, J.P.; Burke, K.; Ernzerhof, M. Generalized gradient approximation made simple. Phys. Rev. Lett. 1996, 77, 3865-3868. [CrossRef]

29. Ernzerhof, M.; Perdew, J.P.; Burke, K. Coupling-constant dependence of atomization energies. Int. J. Quantum Chem. 1997, 64, 285-295. [CrossRef]

30. Momma, K.; Izumi, F. VESTA 3 for three-dimensional visualization of crystal, volumetric and morphology data. J. Appl. Crystallogr. 2011, 44, 1272-1276. [CrossRef]

31. Gajdoš, M.; Hummer, K.; Kresse, G.; Furthmüller, J.; Bechstedt, F. Linear optical properties in the projector-augmented wave methodology. Phys. Rev. B-Condens. Matter Mater. Phys. 2006, 73, 045112. [CrossRef]

32. Wang, V.; Xu, N.; Liu, J.-C.; Tang, G.; Geng, W.T. VASPKIT: A Pre-and Post-Processing Program for VASP code. Comp. Phys. Commun. 2021, 267, 108033. [CrossRef]

33. Heyd, J.; Scuseria, G.E.; Ernzerhof, M. Hybrid functionals based on a screened Coulomb potential. J. Chem. Phys. 2003, 118, 8207-8215. [CrossRef]

34. Gill, S.K.; Huang, J.; Mausz, J.; Gakhar, R.; Roy, S.; Vila, F.; Topsakal, M.; Phillips, W.C.; Layne, B.; Frenkel, A.I.; et al. Connections between the Speciation and Solubility of $\mathrm{Ni}(\mathrm{II})$ and $\mathrm{Co}(\mathrm{II})$ in $\mathrm{Molten} \mathrm{ZnCl}_{2}$. J. Phys. Chem. B 2020, 124, 1253-1258. [CrossRef] [PubMed]

35. Greenberg, J.; Sundheim, B.R. Absorption spectra in molten salt solutions. J. Chem. Phys. 1958, 29, 1029-1032. [CrossRef]

36. Sundheim, R.; Harrington, G. Absorption spectrum of $\mathrm{NiCl}_{2}$ in molten $\mathrm{LiCl} / \mathrm{KCl}$. J. Chem. Phys. 1959, 31, 700-701. [CrossRef]

37. Gabriel, J.; Vincent, D.; Bouteillon, J.; Poignet, J.; Volkovich, V.; Griffiths, T. Molybdenum chemistry in molten LiCl-KCl eutectic: An electrochemical and absorption spectroscopy study of the concentration dependent stability of solutions of $\mathrm{K}_{3} \mathrm{MoCl}_{6}$. Electrochim. Acta 1999, 44, 4619-4629. [CrossRef]

38. Brevnova, N.P.; Polovov, I.B.; Chernyshov, M.V.; Volkovich, V.A.; Vasin, B.D.; Griffiths, T.R. Electronic Absorption Spectra of Niobium Species in Halide Melts. ECS Trans. 2013, 50, 325-338. [CrossRef] 